\title{
FREQUENCY VERSUS TIME DOMAIN PARAMETER ESTIMATION: APPLICATION TO A SLOT MILLING OPERATION
}

\author{
O. TURKAY \\ Department of Mechanical Engineering, Bogazici University, Bebek, Istanbul, Turkey \\ AND \\ A. G. UlsoY \\ Department of Mechanical Engineering, G. G. Brown Building, University of Michigan, \\ Ann Arbor, MI 48109, U.S.A.
}

(Received April 1987, accepted April 1988)

\begin{abstract}
The time domain recursive least squares estimation of parameters in the presence of additive output noise of harmonic form leads to biased estimates. However, frequency domain least squares parameter estimation, where the frequency range contaminated by the noise is eliminated, can be used to obtain good parameter estimates. Both the time domain and frequency domain methods are described, and applied to the example of a slot milling operation. A model of the resultant force response to feedrate changes in slot milling, based on previously reported experimental studies, is presented. The force measurement is corrupted by harmonic noise arising from runout on the milling cutter. Simulation studies are performed, using a flat band width multi-frequency test signal to persistently excite the system. The time domain approach leads to poor estimates as expected, while the frequency domain approach gives good parameter estimates. The advantages and disadvantages of both methods are discussed.
\end{abstract}

\section{INTRODUCTION}

This paper considers time domain vs. frequency domain parameter estimation in linear systems with additive harmonic output noise. The study was motivated by the particular problem of adaptive control in a slot milling operation as described below, and this example is used as a basis for comparing the time domain vs. frequency domain parameter estimation approaches. The conclusions and results, however, should be relevant to a wide variety of parameter estimation problems with additive harmonic output noise.

Numerically controlled (NC) and computer numerically controlled (CNC) machine tools are used extensively for machining operations to reduce operator input, resulting in significant improvements in productivity. However, further improvements in metal removal rates, and in the tool life can be achieved by on-line manipulation of the feeds and speeds [1], [2]. This can, for example, be achieved by using a process controller where the resultant cutting force can be measured and fed back to manipulate the feed in order to maintain a constant, optimum, force level. Such process control strategies have generated considerable research interest in recent years, particularly in light of difficulties due to process parameter variations [2]-[5]. Adaptive controllers, which combine on-line parameter estimation and control [6]-[8], have been proposed and implemented to address these difficulties [3]-[5], [9], [10].

In adaptive control of the force during a milling operation, difficulties in parameter estimation arise due to runout noise [4]. The runout noise comes from small geometric 
imperfections in the cutter, as well as from small imperfections in the mounting of the cutter in the chuck. Thus, it occurs at a frequency given by the number of teeth on the cutter times the spindle rotational frequency. The harmonic nature of the noise necessitates a recursive parameter estimation algorithm with some form of prefiltering, and a stability analysis or check is required. Also the runout noise model contains roots on the unit circle, and leads to problems with unknown initial conditions whose influence does not decay [6], [8]. If this runout noise frequency is sufficiently high, it can be effectively filtered to eliminate problems of bias in the parameter estimates [10]. However, at lower spindle speeds the runout noise frequency spectrum may begin to overlap with the process model spectrum, necessitating the inclusion of the filter dynamics in the process model and leading to a parameter estimation problem with a potentially large number of unknown parameters [10]. Thus, a high order model may be required as the basis for the adaptive controller design, the model may depend on the filter as well as process dynamic characteristics, and the resulting adaptive controller may require a large computation time between samples.

Braun and Seth [11] have suggested the use of frequency domain filtering techniques for rotating machinery. Such an approach is based on using a discrete Fourier transform (DFT) of a time domain signal, removing from the spectrum the information at the rotational speed frequency (and its multiples), then using an inverse DFT to obtain the filtered time domain signal. A similar approach has been successfully used in refs. [12] and [13] for estimating the oil-film coefficients in a rotor-bearing system. This method uses a least squares procedure to directly estimate the parameters in the frequency domain. Because of its frequency domain nature, it has the inherent capability of filtering measurement noise at known frequencies.

The purpose of this paper is to carry out a quantitative comparison of the time domain and frequency domain parameter estimation methods, for the particular example of the force response model in milling including the runout noise. First, the milling force response model used as the basis for the simulation studies is presented. Next, the time domain and frequency domain parameter estimation methods are described. Simulation results, showing and comparing the performance of these two methods, are also presented. The results show the poor performance of the time domain least squares parameter estimation in the presence of runout noise, and the good performance obtained using the frequency domain approach. However, the frequency domain approach is not recursive, and further work will be needed to make it an attractive on-line estimation method.

\section{MILLING PROCESS MODEL}

Experiments have been performed to develop a dynamic model of the resultant force $\left(F_{R}\right)$ response in slot milling to changes in the feedrate $(f)$. The experiments are described in detail in ref. [4], and briefly summarised here.

A CNC milling machine was instrumented with a three component force dynamometer, and an analog circuit to compute the resultant force

$$
F_{R}=\left(F_{x}^{2}+F_{y}^{2}+F_{z}^{2}\right)^{1 / 2}
$$

during a slot milling operation. A laboratory minicomputer system was used to provide a step change in the feedrate during the milling operation, and to sample and collect the feedrate and resultant force data. A series of cutting tests were performed at different depths of cut $(a)$, spindle rotational speeds $\left(N_{r}\right)$, and feedrates $(f)$. These tests, together with a simple mechanistic model of the cutter defiections, show that the force response 
to feedrate changes in the slot milling process can be suitably modeled by the following non-linear differential equation [4]

$$
\ddot{F}_{R}+2 \zeta \omega_{n} \dot{F}_{R}+\omega_{n}^{2} F_{R}=K_{s} a^{\beta} \omega_{n}^{2} f^{\alpha}+K_{s} a^{\beta} 2 \zeta \omega_{n} \dot{f}^{\alpha} .
$$

Note that at steady state the above equation reduces to a form commonly employed in the manufacturing literature [1],

$$
F_{R}=K_{s} a^{\beta} f^{\alpha}
$$

and $K_{s}$ is referred to as the specific cutting force coefficient.

The non-linear equation (2) can be linearised about a steady state operating point using a Taylor series expansion. Defining the incremental variables $\Delta F_{R}$ and $\Delta f$ as

$$
\begin{gathered}
F_{R}=F_{R 0}+\Delta F_{R} \\
f=f_{0}+\Delta f
\end{gathered}
$$

and neglecting higher order terms in the Taylor series, we get the linear equation

$$
\Delta \ddot{F}_{R}+2 \zeta \omega_{n} \Delta \dot{F}_{R}+\omega_{n}^{2} \Delta F_{R}=\left(K_{s} a^{\beta} \alpha f_{0}^{\alpha-1} \omega_{n}^{2}\right)\left(\Delta f+\left(2 \zeta / \omega_{n}\right) \Delta \dot{f}\right) .
$$

Equation (5) can be written in transfer function form as

$$
\frac{\Delta F_{R}(s)}{\Delta f(s)}=\frac{\left(c_{3}+c_{4} s\right)}{\left(s^{2}+c_{1} s+c_{2}\right)}
$$

where

$$
\begin{gathered}
c_{1}=2 \zeta \omega_{n} \\
c_{2}=\omega_{n}^{2} \\
c_{3}=K_{s} a^{\beta} \alpha f_{0}^{\alpha-1} \omega_{n}^{2} \\
c_{4}=K_{s} a^{\beta} \alpha f_{0}^{\alpha-1} 2 \zeta \omega_{n} .
\end{gathered}
$$

Rewriting the non-linear equation (2) in state variable form gives

$$
\begin{gathered}
\dot{x}_{1}=-c_{1} x_{1}+x_{2}+\left(K_{s} a^{\beta} c_{1}\right) u^{\alpha} \\
\dot{x_{2}}=-c_{2} x_{1}+\left(K_{s} a^{\beta} c_{2}\right) u^{\alpha}
\end{gathered}
$$

where $x_{1}=F_{R}$ and $u=f$. To account for the runout noise $d(t)$, the output equation is written as,

$$
y=x_{1}(t)+d(t)=F_{R}+d_{0} \sin \omega_{d} t
$$

where $\omega_{d}=2 \pi N_{t}\left(N_{r} / 60\right) \mathrm{rad} / \mathrm{sec}$ is the runout noise frequency, and $d_{0}$ is the runout noise amplitude. The linear equations (5) or (6) are used as the basis for the parameter estimation methods described below. The non-linear equation (8) is used in the simulations, with equation (9), to generate the data used in the parameter estimation study. Typical values of the process parameters are obtained from [4], and the standard values used in the simulation studies are summarised in Table 1. 
TABLE 1

Standard values of the process parameters used in the simulation studies

\begin{tabular}{ll}
\hline \multicolumn{1}{c}{ Parameter } & Standard value used in simulation \\
\hline$f_{0}$, nominal feedrate & $0 \cdot 847 \mathrm{~mm} / \mathrm{sec}$ \\
$\alpha$, feedrate exponent & $0 \cdot 73$ \\
$\beta$, depth exponent & $1 \cdot 4$ \\
$K_{s}$, specific cutting force coefficient & $150 \cdot 0$ \\
$N_{r}$, spindle speed & $575 \cdot 0$ or $200 \cdot 0 \mathrm{rpm}$ \\
$N_{t}$, number of teeth & 4 or 2 \\
$d_{0}$, runout noise peak to peak magnitude & $25 \cdot 0$ or $50.0 \mathrm{~N}$ \\
$\zeta$, process damping ratio & $0 \cdot 55$ \\
$\omega_{n}$, process natural frequency & $3 \cdot 0 \mathrm{rad} / \mathrm{sec}$ \\
$a$, depth of cut & $3.0 \mathrm{~mm}$ \\
\hline
\end{tabular}

\section{PARAMETER ESTIMATION METHODS}

The goal is to estimate the parameters of the linear model presented in the previous section from input $(\Delta f)$ and output $\left(\Delta F_{R}\right)$ measurements. In particular it is desired to investigate the effect of the runout noise on both time domain and frequency domain least squares parameter estimates. First the choice of the input signal $(\Delta f)$ for estimation is discussed, then both the time domain and frequency domain least squares parameter estimation methods are outlined.

For convergence of the parameter estimates to the true parameter values, it is well known that the input signal must be sufficiently rich and persistently exciting [6], [8]. For the model presented above a persistently exciting input signal with a minimum of two distinct frequencies within the bandwidth of the system is required. There are several candidates for such an input signal, and the pseudo-random binary sequence (PRBS) is one of the most commonly used. Here the Schroeder-phased harmonic sequence (SPHS) is used [14].

SPHS is a periodic signal which can be constructed using the formula

$$
f(t)=\sum_{k=1}^{N_{h}}\left(p_{k} / 2\right)^{1 / 2} \cos \left(k \omega_{0} t+\Theta_{k}\right)
$$

where $p_{k}$, with $\sum_{k=1}^{N_{h}} p_{k}=1$, is the relative power and $\Theta_{k}$ is the phase angle of the $k$ th harmonic. $N_{h}$ is the number of harmonics, and $\omega_{0}$ is the fundamental frequency of the SPHS. The SPHS can be synthesised to give any arbitrarily defined spectrum, including a flat modulus spectrum with a sharp cut off. This is not possible with PRBS which always has the same shape of modulus spectrum and possesses parasitic frequencies beyond its flat bandwidth. The SPHS is a low peak factor signal and persistently excites all of the system modes without violating the linear operation condition. By a low peak factor signal, we mean that the difference between the minimum and maximum amplitudes of the signal divided by its root mean square value is low. This can be achieved by using the following recursive formula to compute the phase angles $\Theta_{k}$ in equation (10), [14]

$$
\Theta_{k}=\Theta_{k-1}-2 \pi \sum_{i=1}^{k-1} p_{i} ; \quad k=1,2, \ldots, N_{h} .
$$

\subsection{TIME DOMAIN ESTIMATION}

The time domain least squares parameter estimation method, requires a difference equation form of the linear equation (5)

$$
\Delta F_{R}(k)=-a_{1} \Delta F_{R}(k-1)-a_{2} \Delta F_{R}(k-2)+b_{0} \Delta f(k-1)+b_{1} \Delta f(k-2)+e(k)
$$


where $k$ represents the sampling instant (i.e. $t_{k}=k \Delta t$, where $\Delta t$ is the sampling period) and the parameters in equation (12) are related to those in equation (6) by

$$
\begin{gathered}
a_{1}=2 \exp \left(-c_{1} \Delta t / 2\right) \cos \left(\omega^{\prime} \Delta t\right) \\
a_{2}=\exp \left(-c_{1} \Delta t\right) \\
b_{0}=\left(c_{3} / c_{2}\right)\left(1-\exp \left(-c_{1} \Delta t / 2\right) \cos \left(\omega^{\prime} \Delta t\right)+\left(\zeta \omega_{n} / \omega^{\prime}\right) \sin \left(\omega^{\prime} \Delta t\right)\right) \\
+\left(c_{4} / \omega^{\prime}\right) \exp \left(-c_{1} \Delta t / 2\right) \sin \left(\omega^{\prime} \Delta t\right) \\
b_{1}=\left(c_{3} / c_{2}\right) \exp \left(-c_{1} \Delta t / 2\right)\left(\exp \left(-c_{1} \Delta t / 2\right)+\left(\zeta \omega_{n} / \omega^{\prime}\right) \sin \left(\omega^{\prime} \Delta t\right)-\cos \left(\omega^{\prime} \Delta t\right)\right) \\
-\left(c_{4} / \omega^{\prime}\right) \exp \left(-c_{1} \Delta t / 2\right) \sin \left(\omega^{\prime} \Delta t\right)
\end{gathered}
$$

where $\omega^{\prime}=\omega_{n}\left(1-\zeta^{2}\right)^{1 / 2}$. Equation (12) can be written in regression form

$$
\Delta F_{R}(k)=\left[-a_{1}-a_{2} b_{0} b_{1}\right]\left[\begin{array}{c}
\Delta F_{R}(k-1) \\
\Delta F_{R}(k-2) \\
\Delta f(k-1) \\
\Delta f(k-2)
\end{array}\right]+e(k)=\Theta^{T} \phi(k-1)+e(k)
$$

where $\Theta$ is the parameter vector, and $\phi(k)$ is the measurement vector at the $k$ th sampling instant. The least squares parameter estimate is given by [6], [8]

$$
\hat{\boldsymbol{\Theta}}(N)=\left[\sum_{i=1}^{N} \phi(i-1) \phi(i-1)^{T}\right]^{-1}\left[\sum_{i=1}^{N} \phi(i-1) \Delta F_{R}(i)\right] .
$$

If the input $\Delta f(k)$ is sufficiently rich and persistently exciting, and the measurement noise $e(k)$ is zero mean and Gaussian, then the parameter estimate converges to the true value of the parameter vector. With the sinusoidal noise term in equation (9), the parameter estimates can be expected to be biased [6], [8].

Equation (15) represents the off-line least squares estimate, and a recursive form of equation (15), suitable for on-line estimation, can also be employed as was done in ref. [10]. Here we use the off-line version for purposes of direct comparison with the frequency domain approach described below.

\subsection{FREQUENCY DOMAIN ESTIMATION}

If a multifrequency feedrate signal is used to produce small perturbations in the resultant cutting force about an operating point, then equation (6) can be written in the frequency domain as:

$$
\left(-\omega^{2}+c_{1} j \omega+c_{2}\right) \Delta F_{R}(j \omega)=\left(c_{3}+c_{4} j \omega\right) \Delta f(j \omega)
$$

Letting

$$
G(j \omega)=\frac{\Delta F_{R}(j \omega)}{\Delta f(j \omega)}=G^{R}+j G^{I}
$$

then we have

$$
\left(-\omega^{2}+c_{1} j \omega+c_{2}\right)\left[G^{R}+j G^{I}\right]=\left(c_{3}+c_{4} j \omega\right) .
$$


Equation (16) can be separated into its real and imaginary parts and then put in matrix form as

$$
\left[\begin{array}{cccc}
-\left(n \omega_{0}\right)\left(G^{I}\right) n & \left(G^{R}\right) n & -1 & 0 \\
\left(n \omega_{0}\right)\left(G^{R}\right) n & \left(G^{I}\right) n & 0 & \left(n \omega_{0}\right) \\
\vdots & \vdots & \vdots & \vdots
\end{array}\right]\left[\begin{array}{c}
c_{1} \\
c_{2} \\
c_{3} \\
c_{4}
\end{array}\right]=\left[\begin{array}{cc} 
& \cdot \\
\left(n \omega_{0}\right)^{2} & \left(G^{R}\right) n \\
\left(n \omega_{0}\right)^{2} & \left(G^{l}\right) n \\
\vdots & \vdots
\end{array}\right]
$$

where $n=1,2,3, \ldots, N$ and $\omega=n \omega_{0}$.

Equation (18) can be represented in standard form as,

$$
\mathbf{X C}=\mathbf{Y}
$$

where the $2 N \times 4$ matrix $X$ and the $2 N \times 1$ vector $Y$ are formed from frequency domain measurements. The $4 \times 1$ vector $C$ is the matrix of unknown coefficients to be estimated.

The least-squares solution of equation (19) is given by [15]

$$
\hat{\mathrm{C}}=\left(\mathbf{X}^{T} \mathbf{X}\right)^{-1} \mathbf{X}^{T} \mathbf{Y}
$$

Any particular frequency range can be excluded from the estimation by simply deleting the corresponding rows in $\mathbf{X}$ and $\mathbf{Y}$ in equation (18). This property is useful for filtering any measurement noise within the band limit given by $N \omega_{0}$. The noise beyond this limit in particular the runout noise is automatically filtered by equation (18).

The frequency-domain parameter estimation method can be summarised as follows

(i) Apply the FFT algorithm to signals $\Delta F_{R}(k)$ and $\Delta f(k)$ where $k$ denotes the sampled forms of $\Delta F_{R}(t)$ and $\Delta f(t)$ respectively.

(ii) Fill the matrices $\mathbf{X}$ and $\mathbf{Y}$ with the frequency response coefficients obtained by equation (19).

(iii) Use equation (20) to obtain the estimates for $c_{1}, c_{2}, c_{3}$ and $c_{4}$.

In order to evaluate the estimates and the estimation procedure quantitatively, statistical results such as sum of the squares of the residuals, standard errors significance test and confidence intervals can be computed with little extra effort [15].

The sum of the squares of the residuals is given by,

$$
S=\hat{\mathbf{E}}^{T} \hat{\mathbf{E}}
$$

where $\hat{\mathbf{E}}$ is the residual vector given by

$$
\hat{\mathbf{E}}=\mathbf{Y}-\hat{\mathbf{Y}}=\mathbf{Y}-\mathbf{X} \hat{\mathbf{C}}
$$

and $\hat{\mathbf{Y}}$ is the vector containing the estimated values of the elements in $\mathbf{Y}$. Standard errors of the estimated coefficients can be obtained from

$$
s \cdot e \cdot\left(\hat{c}_{i}\right)=W \sqrt{i \text { th diagonal of }\left(\mathbf{X}^{T} \mathbf{X}\right)^{-1}}
$$

where $\hat{c}_{i}$ is the $(i)$ th element of $\hat{\mathbf{C}}$ and

$$
W=\frac{1}{2 N} \hat{\mathbf{E}}^{T} \mathbf{E}
$$

Standard errors are used to test the significance of each coefficient. It is possible that there are some parameters that have negligible effect on the system response despite the high goodness of fit value. In statistical terms, the hypothesis to be tested is $\hat{c}_{i}=0$. Standard 
$t$-tests can be used for this purpose. The estimated coefficient $\hat{c}_{i}$ is not zero with probability of $(1-p)$ if

$$
\frac{\left|\hat{c}_{i}\right|}{s \cdot e\left(\hat{c}_{i}\right)}>t_{D F}, p
$$

where $t_{D F}, p$ is tabulated in $t$-distribution tables for $D F$ degrees of freedom and the probability $p$.

It is also possible to calculate the confidence interval of each estimated coefficient

$$
\hat{c}_{i}\left(1 \pm t_{D F}, p \frac{\left|\hat{c}_{i}\right|}{s \cdot e \cdot\left(\hat{c}_{i}\right)}\right) .
$$

If the above region contains zero, then the coefficient is said to be insignificant.

In the following results section the parameter estimates by both frequency and time domain least squares estimation methods are presented for the simulated slot milling operation.

\section{RESULTS AND DISCUSSION}

The equations (8) and (9) with zero initial conditions and $f=f_{0}+\Delta f$ for $t \geqslant 0$ were used to simulate the slot milling process using the parameter values given in Table 1 . The feedrate perturbation $\Delta f$ is simulated using an SPHS with $N_{h}=16$ and $\omega_{0}=1 \cdot 2275$ as shown in Fig. 1. The resultant force response is illustrated in Fig. 2 for $N_{r}=575 \mathrm{rpm}$, $N_{t}=4$, the runout noise $d(t)=50 \sin 240.86 t$, and a sampling period of $\Delta t=0.01 \mathrm{sec}$. The total simulation time is $15 \cdot 35 \mathrm{sec}$ (1536 samples). The modulus spectrum of $\Delta f$ is shown in Fig. 3, and is flat up to the frequency $N_{h} \omega_{0}=19.632 \mathrm{rad} / \mathrm{sec}$. Figure 4 shows the magnitude of the frequency response function $G(j \omega)=\Delta F_{R}(j \omega) / \Delta f(j \omega)$ vs. $\omega$.

For the estimation results shown below, since the linearised equations about the equilibrium are used, the first 1024 data points were discarded and the final 512 data points were used. The frequency domain information was obtained using a fast Fourier transform (FFT) algorithm. The frequency domain results given below use the first 14

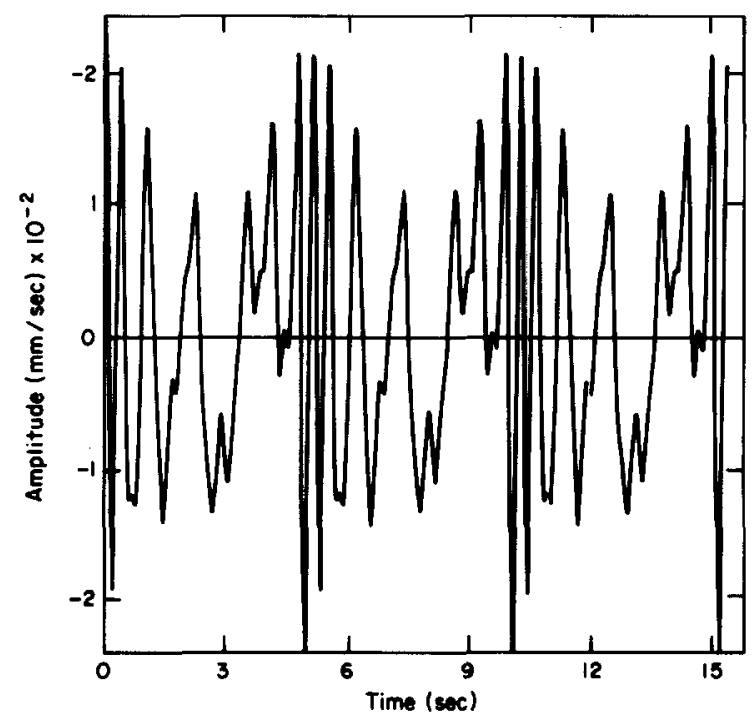

Figure 1. Three periods of SPHS feed perturbation ( $\Delta t=0.01 \mathrm{sec}, 1536 \mathrm{samples})$. 


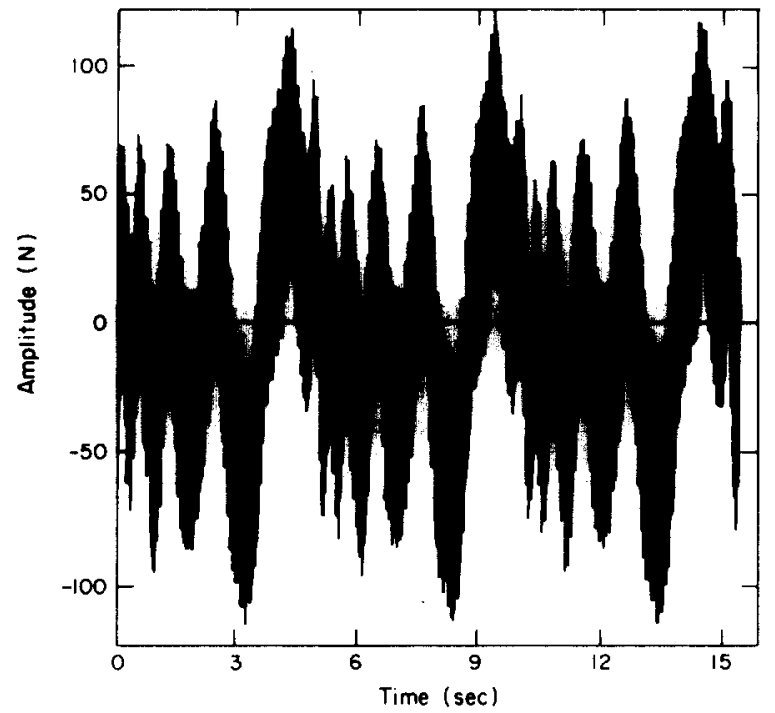

Figure 2. Three periods of the resultant force with noise (noise $d(t)=50 \sin 240.86 t$ ).

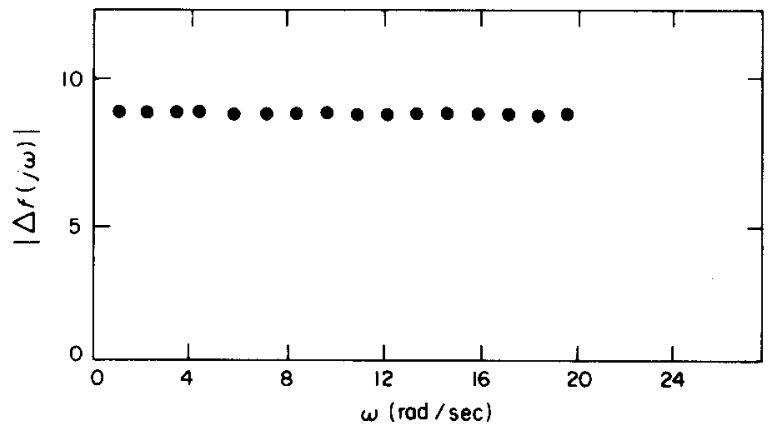

Figure 3. Modulus spectrum of SPHS.

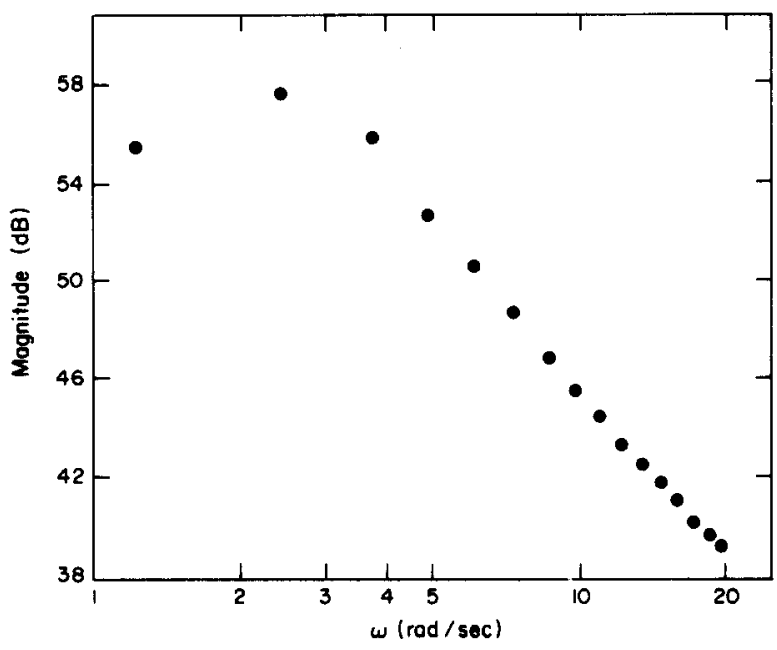

Figure 4. Magnitude of frequency response. 


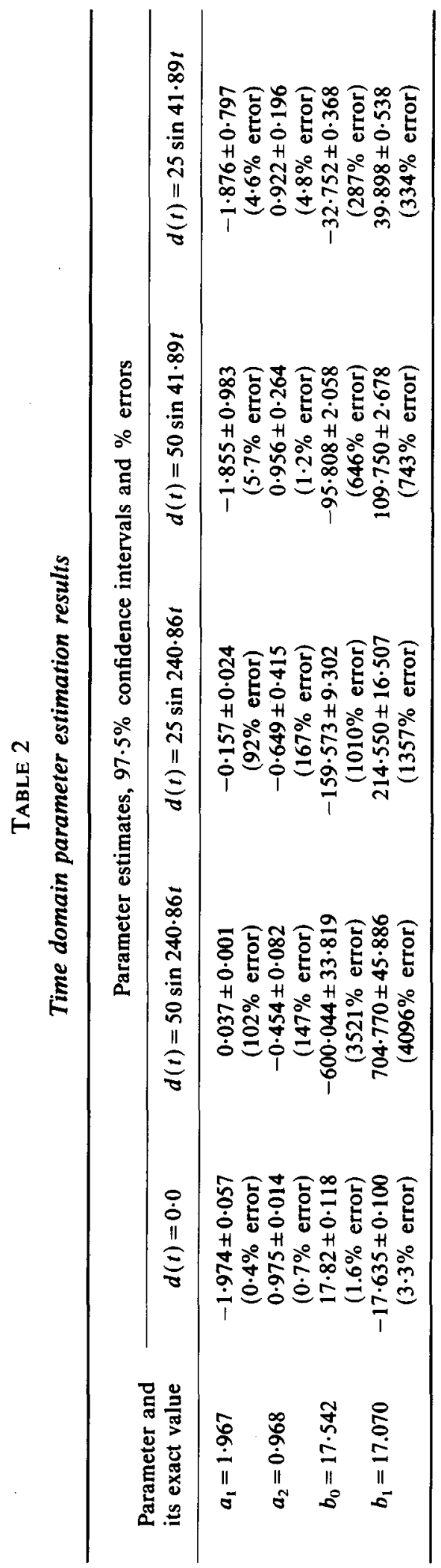




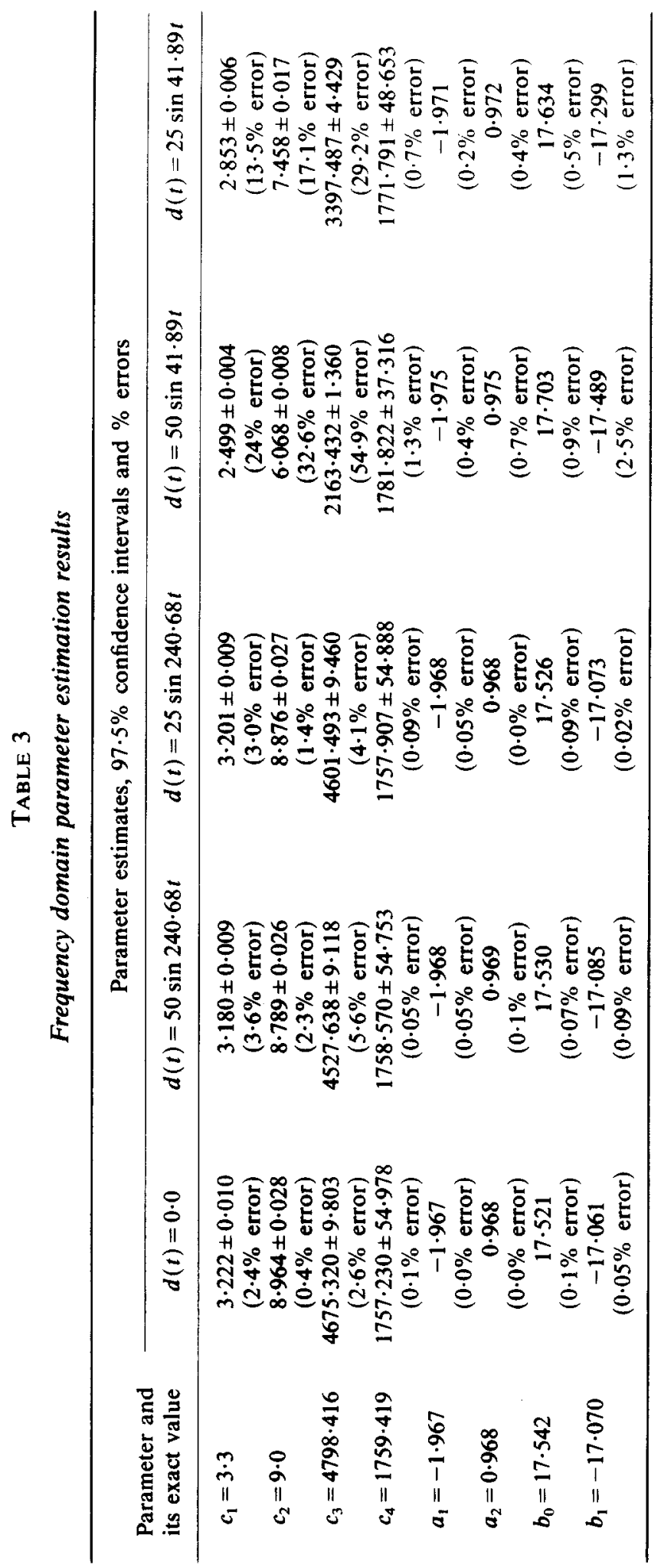


harmonics (i.e. $N=14$ in equation (18)). The numerical integration, FFT and matrix and vector algebra were performed using standard routines available in the International Mathematical and Statistical Library [16]. The programming was done in FORTRAN 77 on a CDC-170/815 computer.

Based on the time domain parameter estimation results in Table 2 one can make the following observations

1. Without runout noise the parameter estimates were excellent. All parameter estimates were significant since the confidence intervals do not include zero, and the maximum error (which is for the parameter $b_{1}$ ) is $3 \cdot 3 \%$.

2. In the presence of runout noise the parameter estimates are unacceptable, with maximum errors of several hundred to several thousand per cent.

3. When the runout noise amplitude is lowered from $d_{0}=50 N$ to $d_{0}=25 N$ the parameter estimates are slightly improved, but still remain unacceptable.

4. The parameter estimates for the lower runout noise frequency $\omega_{d}=41.89 \mathrm{rad} / \mathrm{sec}$ (i.e. $N_{r}=200 \mathrm{rpm}, N_{t}=2$ ) are improved over the $\omega_{d}=240.86 \mathrm{rad} / \mathrm{sec}\left(\right.$ i.e. $N_{r}=575 \mathrm{rpm}$, $\left.N_{t}=4\right)$ case. However, again the parameter estimates are unacceptable in both cases.

Based on the frequency domain parameter estimation results given in Table 3, one can note the following

1. The parameter estimation results are quite good in all cases, even in the presence of the runout noise. When $d(t)=0$, the maximum error, which is for the parameter $c_{3}$, is $2.6 \%$. The confidence intervals do not include zero in any of the cases presented, and so the estimated parameters are significant.

2. When equation (13) is used to calculate $a_{1}, a_{2}, b_{0}$ and $b_{1}$ from the estimated $c_{1}, c_{2}$, $c_{3}$ and $c_{4}$, these estimates never show maximum errors greater than $2.5 \%$. Whereas the maximum errors for the estimated $c_{i}, i=1, \ldots, 4$, can be as large as $54.9 \%$.

3. Although the parameter estimates are acceptable in all cases, some improvement can be observed when the runout noise amplitude is reduced from $d_{0}=50 \mathrm{~N}$ to $d_{0}=25 N$.

4. Unlike the time domain parameter estimation results, the parameter estimation results are somewhat better for the higher runout noise frequency $\omega_{d}=41 \cdot 89 \mathrm{rad} / \mathrm{sec}$ (i.e. $N_{r}=200 \mathrm{rpm}, N_{t}=2$ ).

A comparison of the results in Tables 2 and 3 clearly shows the superiority of the frequency domain estimation method in the presence of runout noise. This is due to the filtering of the information at the runout noise frequency before the least squares problem is solved. The cost associated with this improved performance in parameter estimation, is that an FFT must be performed on the feed and force data. This, with the computational speeds available on current computing hardware, may restrict the applicability of the frequency domain parameter approach to off-line estimation, or on-line estimation of systems with relatively slow dynamics. Note that while both equations (15) and (20) can be rewritten in a recursive form suitable for on-line estimation [6], [8], the FFT required by the frequency domain approach requires a block of $2^{n}$ data points and cannot be performed recursively. The availability of a high speed parallel FFT co-processor would make the frequency domain approach much more attractive for on-line parameter estimation. Note also, that filtering can be used in conjunction with the time domain estimation approach as described in [10]. However, unless there is large separation in frequency between the process dynamics and the runout noise, the filter must also be included in the process model and its parameters estimated along with those of the process. This can lead to a large parameter estimation problem, and significantly increase computation time requirements. 


\section{SUMMARY AND CONCLUSIONS}

The estimation of process model parameters for the resultant force response to feedrate changes in slot milling is made difficult due to the presence of runout noise. A simulation model of this process, based on previous experimental studies, is presented. The simulation studies are used for a quantitative comparison of time domain and frequency domain least squares parameter estimation studies. The frequency domain method uses an FFT and can effectively filter out information associated with the runout noise frequency. Although the particular example of a slot milling operation has been considered here, similar results can be expected for other estimation problems with additive harmonic output noise.

The results show clearly the superiority of the frequency domain parameter estimates in the presence of runout noise. However, the frequency domain approach requires an FFT and may only be suitable for off-line estimation, or on-line estimation of processes with slow dynamics. Further research, and developments in high-speed computing hardware, may make the frequency domain approach more attractive as an on-line estimation method in the future.

\section{ACKNOWLEDGEMENTS}

The authors are pleased to acknowledge the assistance of Hakan Kilitcioglu with the preparation of the plots, and the typing of the manuscript by Bengu Gurtuna.

\section{REFERENCES}

1. Y. KOREN 1984 Computer Control of Manufacturing Systems. New York: McGraw-Hill.

2. A. G. UlsoY, Y. KoREN and F. RASSMUSSEN 1983 ASME Journal of Dynamic Systems, Measurement and Control 105, 107-112. Principal developments in the adaptive control of machine tools.

3. O. MASORY and Y. KOREN 1980 Annals of the CIRP 29, 281-284. Adaptive control system for turning.

4. L. K. Lauderbaugh and A. G. Ulsoy 1985 Presented at the ASME Winter Annual Meeting, Miami Beach, Florida. Dynamic modeling for control of the milling process.

5. M. TomizukA, S. Zhang, T. H. OH and M. S. Chen 1984 Presented at the North American Manufacturing Research Conference, Berkeley, California. Modeling of metal cutting processes for digital control.

6. G. C. GoodWIN and K. S. SIN 1984 Adaptive Filtering Prediction and Control Englewood Cliffs, N.J.: Prentice-Hall.

7. K. J. ASTROM 1983 Automatica 19, 471-486. Theory and applications of adaptive control-a survey.

8. L. LUUNG and T. SODERSTROM 1983 Theory and Practice of Recursive Identification. Cambridge: MIT Press.

9. M. TomizukA and S. ZHANG 1985 Presented at the American Control Conference, Boston, Massachusetts. Modeling and conventional/adaptive PI control of a lathe cutting process.

10. L. K. LAUDERBAugh and A. G. Ulsoy 1986 Presented at the ASME Winter Annual Meeting, Anaheim, California. Model reference adaptive force control in milling.

11. S. G. BRAUN and B. B. SETH 1979 Journal of Sound and Vibration 65, 37-50. On the extraction and filtering of signals acquired from rotating machines.

12. C. R. BURROWS and M. N. SAHINKAYA 1982 ASME Journal of Lubrication Technology 104, 210-215. Frequency domain estimation of linearised oil-film coefficients.

13. M. N. SAHINkAYA, O. S. TURKAY and C. R. BURROWS 1984 ASME Journal of Dynamic Systems, Measurement and Control 106, 342-349. Minimisation of the variance in oil-film damping coefficient estimates.

14. M. R. SCHROEDER 1970 IEEE Transactions on Information and Theory 85-89. Synthesis of low-peak-factor signals and binary sequences with low auto correlation.

15. J. JoHNSTON 1972 Econometric Methods. New York: McGraw Hill.

16. International Mathematical and Statistical Library 1979 IMSL Library Reference Manual, Houston, Texas. 


\section{APPENDIX: NOMENCLATURE}

$a$

$a_{1}, a_{2}, b_{0}, b_{1}$

$c_{1}, c_{2}, c_{3}, c_{4}$

C

$d(t)$

$d_{0}$

$e(k)$

$f(t)$

$F_{R}(t)$

$f_{0}, F_{R O}$

$\Delta f, \Delta F_{R}$

$F_{x}, F_{y}, F_{z}$ $G(j \omega)$

$\left(G^{R}\right)_{n},\left(G^{l}\right)_{n}$

$\boldsymbol{K}_{s}$

$N$

$N_{h}$

$N_{r}$

$N_{t}$

$P_{k}$

$s$

$t$

$\Delta t$

$x_{1}, x_{2}$

$\mathbf{X}$

$\mathbf{Y}$

$\alpha$

$\boldsymbol{\beta}$

$\phi$

$\boldsymbol{\Theta}$

$\omega$

$\omega_{n}$

$\omega_{d}$

$\omega_{0}$

$\omega^{\prime}$

$\zeta$

()$^{T}$

(c)

()$^{\wedge}$

$(-)$

depth of cut, mm

parameters of discrete-time linear model

parameters of continuous-time linear model

parameter vector in frequency domain estimation

runout noise signal, $\mathbf{N}$

amplitude of runout noise, $N$

measurement noise in discrete form

feedrate signal, $\mathrm{mm} / \mathrm{sec}$

resultant force signal, $\mathrm{N}$

stead-state values of feedrate and resultant force respectively

perturbation variables of feedrate and resultant force respectively

force components in cartesian coordinates, $\mathrm{N}$

frequency response function

real and imaginary parts of $G\left(j n \omega_{0}\right)$

specific cutting force coefficient

number of equations in the least squares problem

number of harmonics in the SPHS signal

spindle rotational speed, $\mathrm{rpm}$

number of teeth on the milling cutter

relative power in the $k$ th harmonic

Laplace variable

time

sampling interval

state variables

$2 N \times 4$ measurement matrix in the frequency domain estimation

$2 N \times 1$ measurement vector in the frequency domain estimation

feedrate exponent

depth of cut exponent

measurement vector in the time domain estimation

parameter vector in the time domain estimation

frequency, $\mathrm{rad} / \mathrm{sec}$

process natural frequency, $\mathrm{rad} / \mathrm{sec}$

runout noise frequency, $\mathrm{rad} / \mathrm{sec}$

fundamental frequency of the SPHS, rad/sec

$=\omega_{n}\left(1-\zeta^{2}\right)^{1 / 2}$

process damping ratio

denotes the transpose

denotes the time derivative

denotes the estimate

denotes the average value 